\title{
From tunneling to contact in a magnetic atom: The non-equilibrium Kondo effect
}

\author{
Deung-Jang Choi, ${ }^{1}$ Paula Abufager ${ }^{2}$ Laurent Limot ${ }^{3}$ and Nicolás Lorente, ${ }^{4}$ \\ ${ }^{1}$ CIC nanoGUNE, Tolosa Hiribidea 78, 20018 Donostia-San Sebastian, Spain and IPCMS, CNRS UMR 7504, \\ Université de Strasbourg, 67034 Strasbour, France \\ ${ }^{2}$ Instituto de Física de Rosario, Consejo Nacional de Investigaciones Científicas y Técnicas (CONICET) \\ and Universidad Nacional de Rosario, Bv. 27 de Febrero 210 BIS, 2000 Rosario, Argentina \\ ${ }^{3}$ IPCMS, CNRS UMR 7504, Université de Strasbourg, 67034 Strasbourg, France \\ ${ }^{4}$ Centro de Física de Materiales CFM/MPC (CSIC-UPV/EHU), Paseo Manuel de Lardizabal 5, 20018 \\ Donostia-San Sebastián, Spain and Donostia International Physics Center (DIPC), Paseo Manuel \\ de Lardizabal 4, 20018 Donostia-San Sebastián, Spain
}

(Received 28 September 2016; accepted 9 December 2016; published online 23 December 2016)

\begin{abstract}
A low-temperature scanning tunneling microscope was employed to study the differential conductance in an atomic junction formed by an adsorbed $\mathrm{Co}$ atom on a $\mathrm{Cu}(100)$ surface and a copper-covered tip. A zero-bias anomaly (ZBA) reveals spin scattering off the Co atom, which is assigned to a Kondo effect. The ZBA exhibits a characteristic asymmetric lineshape when electrons tunnel between tip and sample, while upon the tip-Co contact it symmetrizes and broadens. Through density functional theory calculations and the non-equilibrium non-crossing approximation, we show that the lineshape broadening is mainly a consequence of the additional coupling to the tip, while non-equilibrium effects only modify the large-bias tails of the ZBA. Published by AIP Publishing. [http://dx.doi.org/10.1063/1.4972874]
\end{abstract}

\section{INTRODUCTION}

The Kondo effect has a long story of research, from the anomalous low-temperature conductance of noble metals, ${ }^{1}$ the explanation in terms of spin-flip scattering, ${ }^{2}$ to its study on quantum dots, ${ }^{3,4}$ constrictions, ${ }^{5}$ and extended nanosystems. ${ }^{6}$ The use of the scanning tunneling microscope (STM) has undoubtedly revived the research activity around the Kondo effect. The STM joins unprecedented spatial control of an atomic-scale electrode with high-resolution in energy. In this way, the low-energy scale of the Kondo effect can be studied with subatomic precision in single atoms ${ }^{7-13}$ or molecules. ${ }^{14-16}$

Additionally, STM offers the possibility of creating wellcontrolled atomic point contacts by vertically approaching the tip to an adsorbed atom (adatom hereafter). ${ }^{17}$ This drives the microscope in a new regime, where the tip stops being a nonintrusive probe, to become a part of the system to characterize. The sharp zero-bias anomaly (ZBA) in the differential conductance, which is the spectroscopic manifestation of the Kondo effect, is modified by the strong interaction of the adatom with the tip. ${ }^{18-20}$ The resulting new Kondo system is not just the one of an increased electron bath, but the one in the presence of a sizeable electron flow. Important theoretical efforts have been recently given to the study of this so-called non-equilibrium Kondo regime. ${ }^{21-28}$

A simple-minded picture of non-equilibrium effects would consider two biased Fermi energies that should lead to the appearance of two Kondo peaks and to an enhanced and possibly distorted broadening of the spectral features. A more subtle effect is bias-induced decoherence..$^{22,24-26}$ Indeed, the inequivalent Fermi levels create the situation where closed channels for one electrode can be open channels for the other electrode. Hence, virtual transitions involving the atomic levels become real transitions. This leads to a lifetime of intermediate charged states of the atom and thus to the interruption and hence decoherence of the spin flips, which ultimately eliminates the Kondo effect. Non-equilibrium effects can therefore be expected to profoundly affect the ZBA, but, to date, little is known on how these effects actually modify the ZBA line shape.

To study the above effects, we use a low-temperature STM and form a single-atom contact with a $\mathrm{Co}$ atom on $\mathrm{Cu}(100)$. A ZBA is found, which symmetrizes and broadens as the tip is pushed into contact with the atom. To rationalize this behavior, we performed density functional theory (DFT) calculations and transport calculations. The excellent agreement with the measured conductance over the atom allows us to make a simple Kondo model using the non-crossing approximation (NCA) in non-equilibrium ${ }^{23}$ and unravel the contribution of non-equilibrium effects to the experimental ZBA. We find that in first approximation, the modifications of the ZBA in the contact regime are mainly driven by the increased coupling to the electrodes, while the role of the multiple chemical potentials and non-equilibrium decoherence affects the larger-bias features, i.e., the tails of the ZBA. This study shows that the Kondo temperatures extracted in single-atom contacts ${ }^{19,20}$ reflect the enhanced interaction of the magnetic impurity with its metallic environment.

\section{EXPERIMENT}

We used an ultra-high vacuum $\left(<10^{-10}\right.$ mbar $)$ and low temperature $(4.4 \mathrm{~K})$ STM to measure the differential conductance of $\mathrm{Co}$ on $\mathrm{Cu}(100)$. The $\mathrm{Cu}(100)$ surface, as well as the employed tungsten tips, was cleaned in vacuo by sputter/anneal cycles. The tungsten tips were further prepared by indentation into the surface to cover their apex with 
copper. Cobalt atoms were dosed on the cold $\mathrm{Cu}(100)$ substrate by heating a Co wire ( $99.99 \%$ purity) resulting in a low concentration of $5 \times 10^{-3}$ monolayers, Fig. 1(a).

To monitor how the ZBA changes with tip displacement $z$, conductance versus $z$ curves were first obtained by vertically approaching the tip from above a target cobalt atom. A typical curve is presented in Fig. 1(b). A tip displacement of $z=0$ defines the boundary between the tunneling regime $(z>0)$ and the contact regime $(z<0)$. Prior to and after contact the presence of the tip causes readjustments of the adatom adsorption geometry ${ }^{29,30}$ that we will describe in Sec. III. We find that the contact conductance is $G_{c}=1.04 \pm 0.05$ (in units of $2 e^{2} / h$ ), which demonstrates that the tips employed have a monoatomically sharp apex..$^{31,32}$ The contact geometry has therefore a bottleneck structure comprising a $\mathrm{Cu}$ atom at the tip apex and the $\mathrm{Co}$ adatom on $\mathrm{Cu}(100)$, Fig. 2.

The differential conductance as a function of applied bias shows a characteristic evolution with tip excursion, ${ }^{19,20}$
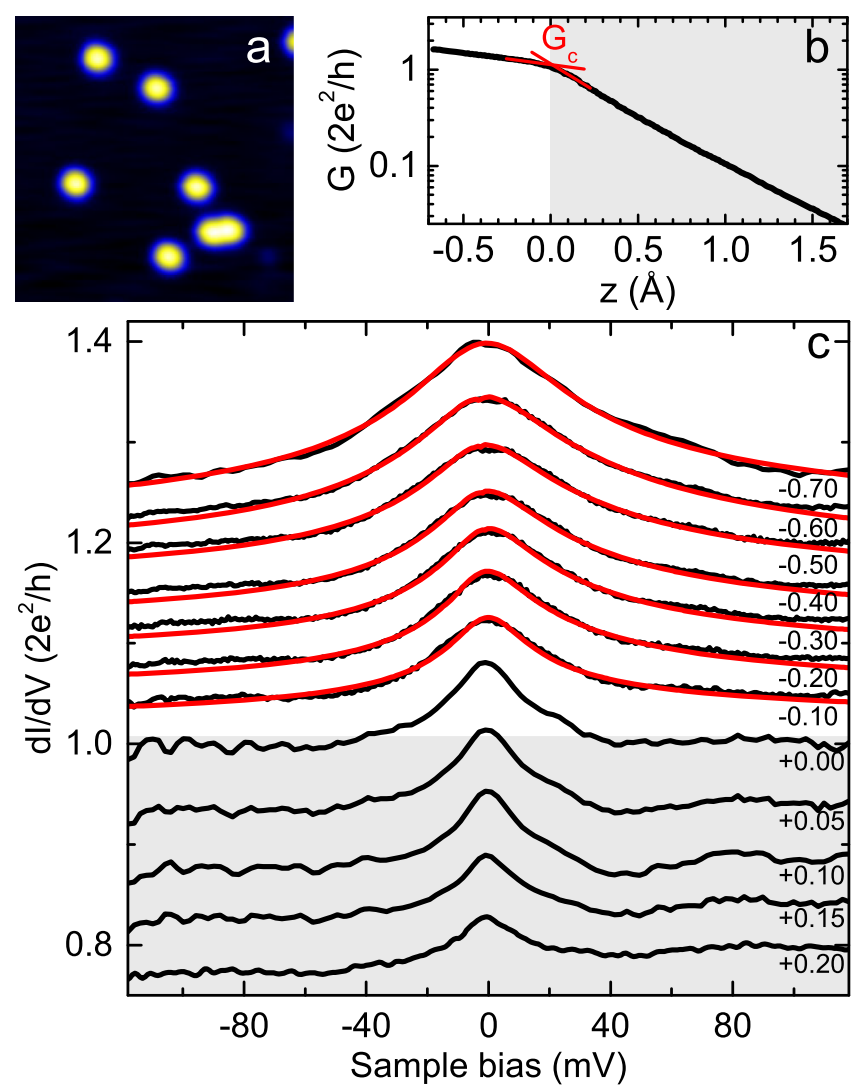

FIG. 1. STM measurements on a Co adatom on $\mathrm{Cu}(100)$. (a) Topographic image of single Co atoms on $\mathrm{Cu}(100)$ (100 pA, $\left.100 \mathrm{mV}, 8 \times 8 \mathrm{~nm}^{2}\right)$. (b) Conductance, $G$, versus $z$ curve above a Co atom; the tunneling regime is indicated by a gray background. The curves are acquired at a fixed bias of $V=-160 \mathrm{mV}$. The contact conductance, $G_{c}$, is extracted by approximating the conductance data in the contact and tunneling regions by straight lines (solid red lines). ${ }^{31}$ Their point of intersection defines $G_{c}$. (c) Set of differential conductance spectra acquired with different tip excursions (indicated in $\AA$ on the right of the panel); the tip was verified to have a flat electronic structure in the bias range presented. Note that a higher tip excursion produces a higher background in the spectrum. The solid red lines correspond to Frota fits. In the tunneling regime, which is indicated by a gray background, the ZBA has a step-like shape. This Fano-like profile results from the interference between tunneling into the Kondo resonance and tunneling directly into the substrate. ${ }^{7,8,10}$ In these experiments, the applied current range varies from 5 to $10 \mu \mathrm{A}$ at $100 \mathrm{mV}$.

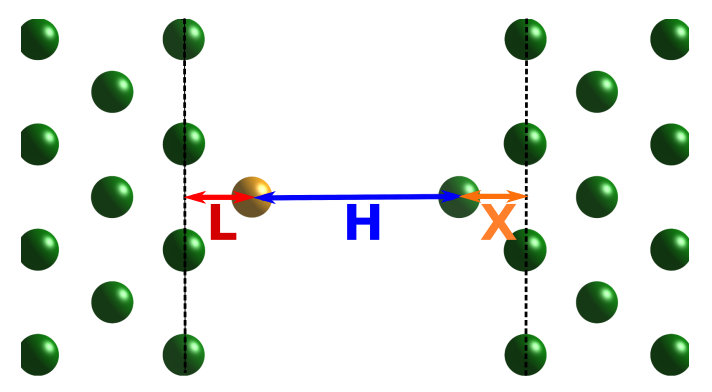

FIG. 2. Scheme of the atomic system computed here. Three main distances are outlined and plotted in Fig. 3 as the electrodes are approached.

Fig. 1(c). The spectra were measured using a lock-in amplifier (modulation: $500 \mu \mathrm{V} \mathrm{rms,} \mathrm{frequency} \mathrm{of} 712 \mathrm{~Hz}$ ) at selected tip excursions above a Co atom. In the tunneling regime, the ZBA exhibits a step-like Fano profile, ${ }^{7,8}$ while in the contact regime, the ZBA develops into a peak, symmetrizes, and broadens with decreasing $z$. At contact, the differential conductance, $G$, of the ZBA as a function of the sample bias, $V$, can be fitted by

$$
G(V)=A+h g(V)
$$

where $h$ and $A$ are constants, and $g$ is the Frota function ${ }^{33}$ given by

$$
g(V)=\operatorname{Re}\left[\sqrt{\frac{i \Gamma}{i \Gamma+e V-\epsilon_{K}}}\right] .
$$

The center of the peak is located at $\epsilon_{K}$ and its broadening is controlled by $\Gamma$, related to the Kondo temperature, $T_{K}$, by ${ }^{12}$

$$
\Gamma=1.455 \times k_{B} T_{K} .
$$

Figure 1(c) shows the excellent fits of the ZBA for different values of $z$ in the contact regime using the above Frota function. We find a monotonic increase of $T_{K}$ from $90 \mathrm{~K}$ at $z=0 \AA$ to $200 \mathrm{~K}$ at $z=-0.7 \AA$, which reflects a larger screening of the magnetic impurity as the tip is pressed into contact with the Co adatom. ${ }^{19,20}$ However, these Kondo temperatures do not include non-equilibrium effects that could change the interpretation of the ZBA broadening. One of the non-equilibrium effects is the population of the junction local modes. Indeed, the population of local vibrational modes of the junction by electron-induced excitation can lead to a change of the $\mathrm{dI} / \mathrm{dV}$ slope.$^{34}$ However, for contact points and tip-adatom junctions, the vibrational dissipation due to bulk-mode excitation is very efficient and the change of slope would be a small contribution to the slopes measured here. ${ }^{34,35}$ Another non-equilibrium effect is the modification of the Kondo properties by the presence of the electron flow. This last one is the object of the present work.

\section{THEORY}

\section{A. Density functional calculations}

Electronic and geometrical structure optimizations have been performed using the spin-polarized generalized gradient approximation (GGA-PBE) ${ }^{36}$ to explore the adsorption of Co atoms on the $\mathrm{Cu}(100)$ surface. To mimic the STM junction, we introduced a second electrode $(\mathrm{a} \mathrm{Cu}(100)$ surface 
with a $\mathrm{Cu}$ adatom, Fig. 2) that accounts for the tip and evaluated the atomic relaxation with VASP ${ }^{37-42}$ and Siesta. ${ }^{43,44}$ The transport calculations have been performed with TranSiesta. ${ }^{45}$

We used a plane wave basis set and the projected augmented wave (PAW) method with an energy cutoff of $400 \mathrm{eV}$. The two surfaces representing substrate and tip were modeled using a slab geometry with a $3 \times 3$ surface unit cell and 5 layers for the surface holding the $\mathrm{Co}$ atom and 6 layers for the tip electrode. The valence-electron wavefunctions were expanded in a basis set of local orbitals in Siesta. A double- $\zeta$ plus polarization (DZP) basis set was used to describe the Co and surface-atom electrons. Diffuse orbitals were used to improve the surface electronic description and a single- $\zeta$ plus polarization (SZP) basis set for the copper electrodes. The use of a DZP basis set to describe the adsorbate states is mandatory in order to yield correct transmission functions. ${ }^{46}$

The k-point sampling was converged at $7 \times 7$, although the sampling was $13 \times 13$ for the transmission calculations. These transport calculations were carried out from firstprinciples with a method based on nonequilibrium Green's functions (NEGFs) combined with DFT as implemented in the TranSiesta package. ${ }^{45}$

Similar calculations using the single-adsorbate KorringaKohn-Rostoker approach were published by the Mertig group. ${ }^{47}$ Our calculations confirmed theirs with some minor difference associated with the particular DFT implementation.

The sequential relaxation of the surface structure as the tip approaches the Co adatom is shown in Fig. 3. When the tip is far from the substrate, the relaxation is monotonous and both the $\mathrm{Co}$ and $\mathrm{Cu}$ adatoms readapt to the shrinking dimensions of the tunneling junction. As the tip approaches, the $d$-electrons of both substrates start to hybridize forming covalent bonds. When the distance of the Co adatom to the surface is roughly
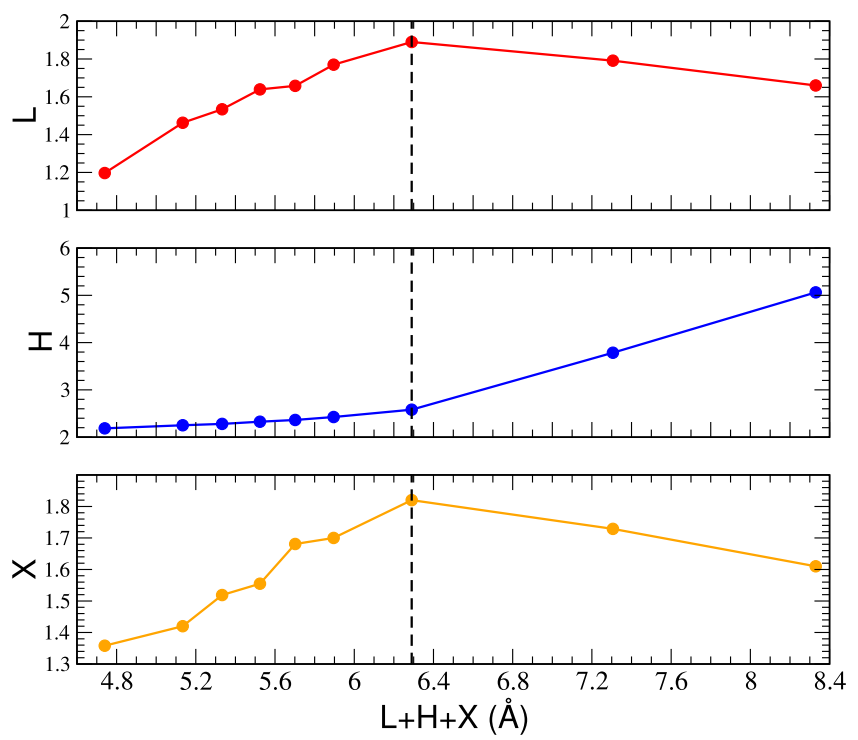

FIG. 3. Geometrical results of the relaxation of a Co adatom as a single-atom $\mathrm{Cu}$ tip is approached. The vertical dashed line denotes the point of contact that corresponds to the formation of an equilibrated covalent bond among all relevant atoms. Following the scheme of Fig. 2, the distance of the Co adatom to the $\mathrm{Cu}(100)$ substrate is denoted as $L$. The tip apex to adsorbate distance is $H$ and $X$ is the distance of the apex $\mathrm{Cu}$ atom to the tip's surface. All distances are in $\AA$. After contact, pressing the tip against the adatom does not lead to a significant reduction of the $\mathrm{Co}-\mathrm{Cu}$ distance $(H)$. the same as the distance of the $\mathrm{Cu}$ tip adatom, there is a sharp transition. The covalent bonds of the Co adatom are roughly as strong with the surface as with the tip. This equilibration of the covalent bonds leads us to define this configuration as the point of contact. Roughly, all evaluated distances behave similarly in each of the two regions that the contact point separates. Before contact, we find that tunneling is a good description of most of the transport features, while after contact important changes happen in the way electrons are transmitted between the tip and the sample. Increasing the tip pressure on the Co adatom leads to pushing the Co adatom to the substrate ( $L$ in Fig. 3), as well as pushing the $\mathrm{Cu}$ adatom to the tip ( $X$ in Fig. 3). Remarkably, the $\mathrm{Co}-\mathrm{Cu}($ tip) distance stays basically constant ( $H$ in Fig. 3).

The two-adatom distance behavior can be understood by studying the interatomic distances as the tip approaches the substrate. Despite reducing the vertical adatom-surface distance, the $\mathrm{Co}-\mathrm{Cu}$ first four adatom is also fairly constant, and the surface $\mathrm{Cu}$ atoms relax laterally yielding space to the $\mathrm{Co}$ adatom. As a consequence, all bonds of the Co adatom with its first five neighbors (including the tip apex) are similar and close to the $\mathrm{Co}-\mathrm{Cu}$ free dimer distance of $2.3 \AA$.

The detailed electronic structure of the Co adatom changes in the contact region. There is a re-ordering of the minority spin $d$-manifold that affects the transmission properties of the adatom when the tip starts interacting with it. However, the overall properties slightly change. The Co adatom can be described as in a $3 d^{8}(\mathrm{~S}=1)$ configuration. This description, corresponding to 2 bohrs magnetons, is maintained even for the shortest tip-surface distances we have evaluated.

In agreement with Ref. 47, we find that transport in the tunneling region basically takes place through the $s p$ electrons of the Co atom. Figure 4 shows a rather exponential decay of the transmission prior to contact. The transmission is due to the majority spin $s p$ electrons, which leads to a positive spin

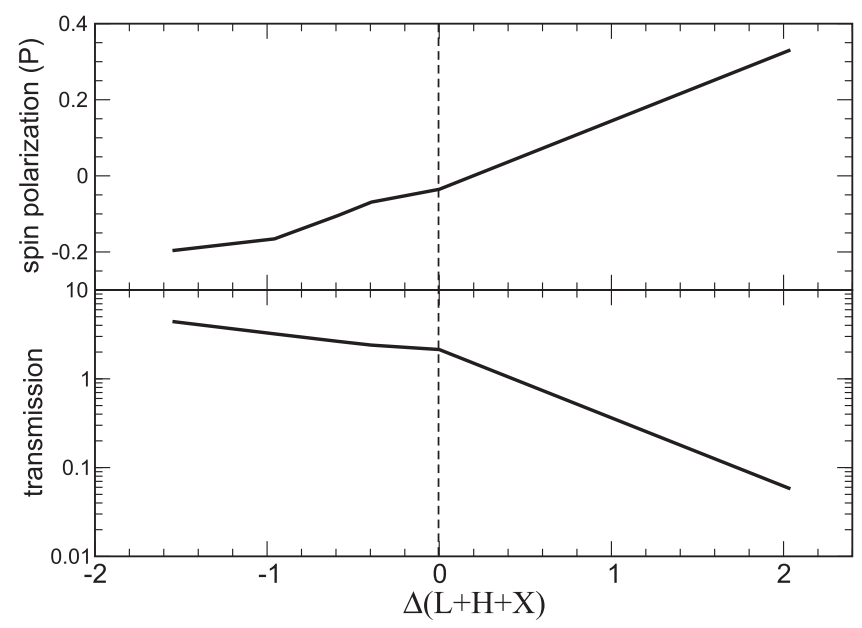

FIG. 4. Spin polarization and electron transmission at the tip-surface distances $\Delta$ (in $\AA$ ), measured from the point of contact that makes it directly comparable with the experimental z, Fig. 1. The spin polarization, Eq. (2), is defined as the spin polarization of the electron transmission, and the total electron transmission is the sum of transmissions for each spin polarization. Again, contact marks a slope change in the transmission function, stabilizing the rise of the transmission as the tip is pressed against the substrate. The spin polarization dramatically changes sign. At contact, it is almost zero and reverses the polarity as the tip is pressed against the substrate. 
polarization as defined by

$$
P=\frac{T_{\uparrow}\left(E_{F}\right)-T_{\downarrow}\left(E_{F}\right)}{T_{\uparrow}\left(E_{F}\right)+T_{\downarrow}\left(E_{F}\right)},
$$

where the transmission per spin $\sigma, T_{\sigma}$, is evaluated at the Fermi energy, $E_{F}$. As the tip approaches, the reorientation and hybridization of the Co electrons with the tip lead to an increase in the $d$-electron contribution to the transmission. As a consequence, the minority spin $d$ electrons dominate the transmission when the system is well into the contact region, and the spin polarization of the current changes sign, Fig. 4. At contact, the electron current becomes unpolarized. This effect is purely due to the opening of the minority spin $d$ channels that compensate the majority spin $s p$ channels, and not to the screening of the Co magnetic moment by the tip. As we said in the previous paragraph, the absolute value of the magnetic moment of the Co adatom is basically constant regardless of the position of the tip. Finally, as the $d$-electrons start to saturate the transmission through the Co adatom, the transmission levels off deep in the contact region, Fig. 4. For shorter distances the transmission will continue increasing due to the direct contribution between $\mathrm{Cu}$ atoms.

The prevalence of minority spins over majority spins in the transmission is clearly seen in Fig. 5. The total transmission is plotted per spin as well as its eigenchannel composition. In the tunneling region, there is a unique eigenchannel that contributes to the current with a large Co $s p$ component. As the tip approaches, the $d$ contribution to the transmission increases, opening two more eigenchannels for the minority spin. The majority spin transmission is too small as can be seen in the left panel of Fig. 5. In the contact region, the transmission is formed by three eigenchannels: the initial one with a large Co $d_{z^{2}}$ component, where the $s p$ component decreases as the tip approaches the substrate, and two more, $d_{x z}$ and $d_{y z}$. Figure 5
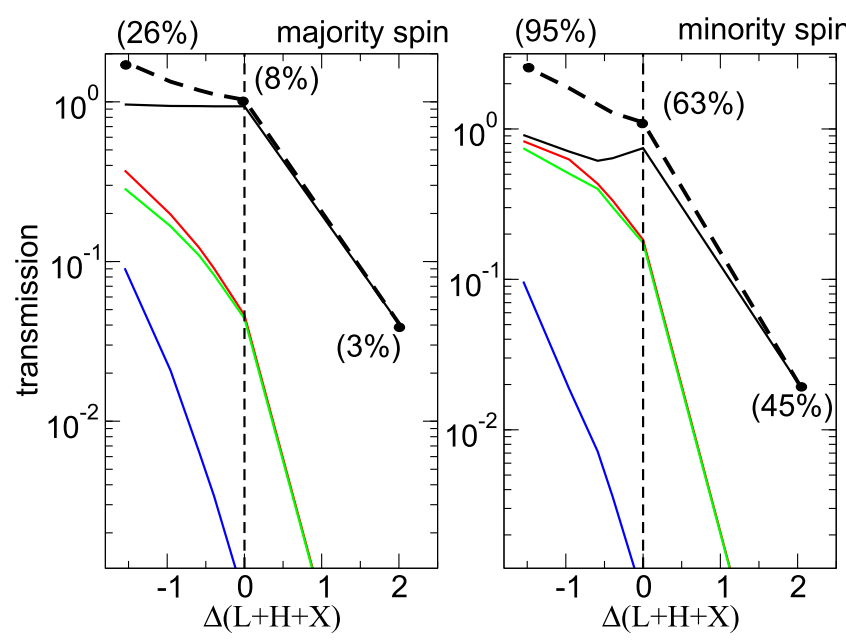

FIG. 5. Transmission per spin at the Fermi energy. The thick dashed line is the total transmission. The other lines correspond to the transmissions at the Fermi energy of the first four eigenchannel components. In the tunneling region, one eigenchannel controls the transmission. In contact, the contribution of the $d$ electrons to transmission leads to the opening of new channels. The nature of the eigenchannels changes as the tip approaches, from a dominating contribution of the $s p$ electrons of cobalt in tunneling to the contribution of $d$ electrons at contact. The values in parentheses are the $d$-electron contribution to the transmission for three selected distances representing the three different transport regions. contains the contribution of $d$ electrons to the transmission for three characteristic tip-substrate distances. We see that the transition to a $d$-dominated conduction takes place for distances shorter than $2 \AA$ from contact. This is in good agreement with our previous experimental studies ${ }^{19}$ where the transition from a Fano lineshape to a peak of the ZBA takes place at about $2 \AA$ from contact. We further discuss this point in the section devoted to Kondo calculations.

The DFT-based transport calculations permit us to conclude that conduction will take place through the $s p$ electrons of the Co adatom in the tunneling regime. In contact, the $d_{z^{2}}$ electrons become dominant and as the tip pressure increases, contribution from the $d_{x z}$ and $d_{y z}$ orbitals becomes important.

\section{B. Non-equilibrium Kondo calculations}

Important recent efforts have been devoted to describing the equilibrium Kondo effect of adsorbed $\mathrm{Co}$ on $\mathrm{Cu}(100)$. Quantum Monte Carlo (CTQMC) calculations ${ }^{48}$ show the need to describe the Kondo effect in this system due to the fluctuations among three different-charge states $\left(d^{7}, d^{8}\right.$, and $d^{9}$ ). They also show how the final details on the orbital degrees of freedom determine the Kondo properties of the system. Numerical renormalization calculations ${ }^{49}$ performed on a model Anderson Hamiltonian obtained by fitting its Hartree-Fock solution to DFT results show the complexity of the problem and the inadequacy of DFT to yield quantitative parameters to reproduce the delicate physics of Kondo impurities. Nevertheless, these calculations give robust insight into the orbital electronic structure at the origin of the Kondo signal of $\mathrm{Co}$ on $\mathrm{Cu}(100)$. Similar results were obtained in Refs. 50 and 51 using the non-crossing and one-crossing approximations to solve model Anderson Hamiltonians of Co on $\mathrm{Cu}(100)$. Other recent works ${ }^{52}$ using CTQMC on DFTfitted Anderson Hamiltonians permit us to rationalize the evolution of Kondo impurities on different metallic substrates.

The above works together with our previous DFT results conclude on characterizing $\mathrm{Co}$ on $\mathrm{Cu}(100)$ as a $\mathrm{S}=1$ system, where the $d_{z^{2}}$ orbital acquires a special meaning due to its enhanced hybridization both to substrate and tip. Our transport calculations in Sec. III A however show that in the tunneling regime, the $s p$-based structure of the Co adatom dominates the transmission, only when the tip is close enough, does the $d_{z^{2}}$ orbital take the lead. This has important consequences for the differential conductance of the Kondo system. As shown in Refs. 49 and 51, Fano profiles develop due to the interference of transmission paths over the atom. Particularly Frank and $\mathrm{Jacob}^{51}$ show that the Co $s$ and $d_{z^{2}}$ electrons interfere in ways that can explain the Fano structures experimentally found in Co on $\mathrm{Cu}(100) .{ }^{19}$ This picture agrees with our transport findings.

In Section III A, we defined the contact point when the $\mathrm{Co}-\mathrm{Cu}$ distances become equal for each of the first four $\mathrm{Cu}$ atoms of the surface and for the tip apex atom. In this case, the couplings $\Gamma_{t}$ and $\Gamma_{s}$ defining the hybridization of the Co orbitals with both electrodes, tip $(t)$ and surface $(s)$, become equal. This is justified by the calculation of the elastic transmission reaching the value of one that can be attained only if both electronic couplings become equal for a Breit-Wigner resonance coupled to two continua. Of course, these couplings 
are very different in the tunneling regime. We evaluate the differential conductance by computing the numerical derivative of the Landauer current, $I$, with respect to applied bias, $V$. Following the above works, ${ }^{49-51}$ we take the conductance to be dominated by the $d_{z^{2}}$, and hybridization functions $\Gamma_{t}$ and $\Gamma_{s}$ that are mutually proportional. In this case, the many-body Landauer current becomes ${ }^{53}$

$$
I=\frac{2 e}{\hbar} \frac{\Gamma_{t} \Gamma_{s}}{\Gamma_{t}+\Gamma_{s}} \int\left[f_{t}(\omega, V)-f_{s}(\omega, V)\right] \rho(\omega, V) d \omega,
$$

where $f_{t}(\omega, V)$ and $f_{s}(\omega, V)$ are the equilibrium Fermi distribution functions for each electrode, with their respective Fermi energies shifted by $\mathrm{eV}$, and $\rho(\omega, V)$ is the non-equilibrium many-body spectral function of the $d_{z^{2}}$ orbital, also known as the non-equilibrium projected density of states on the $d_{z^{2}}$ orbital.

To calculate the non-equilibrium many-body spectral function, we resort to the non-crossing approximation (NCA) where it is particularly simple to include non-equilibrium effects. $^{23,27}$ For this, we use our previous formulation of $\mathrm{NCA}^{54}$ extending hybridization functions to include several electrodes following the equations of Ref. 23. We have assumed a bandwidth of $5 \mathrm{eV}$ where the hybridization function is completely contained.

We first take $\Gamma_{t}=30 \times \Gamma_{s}$. This corresponds to the tunneling regime, where the atom is bound to the surface and follows its density of states as the tip is biased with respect to the surface. Our DFT calculations show that transport takes place through the $s p$ orbitals of the Co atom. To simplify, we assume that there is a single orbital involved, the $d_{z^{2}}$, because we are trying to characterize the evolution of the differential conductance with bias. For this we simply notice that the adatom spectral function is constant for all applied biases, see Fig. 6, and that the final differential conductance is simply proportional to the spectral function, Fig. 7. In this situation, the differential conductance would reflect the ZBA modified by the interference among conduction channels giving rise to the Fano profiles. ${ }^{49,51}$ By fitting a Fano profile, the values of the Kondo temperature can be estimated as well as other properties of the Kondo state. ${ }^{19}$

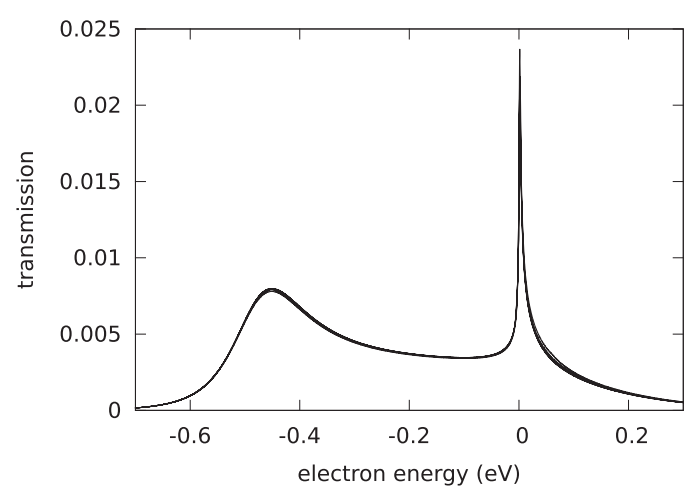

FIG. 6. Transmission curves as a function of the electron energy for 10 different biases applied between tip and substrate between $-0.6 \mathrm{~V}$ and $0.2 \mathrm{~V}$. The 10 transmission curves are basically indistinguishable showing no bias dependence. The coupling of the tip to the Co atom $d_{z^{2}}$ is taken to be 30 times smaller than the Co-substrate coupling. The transmission is directly proportional to the spectral function in the plotted energy range.

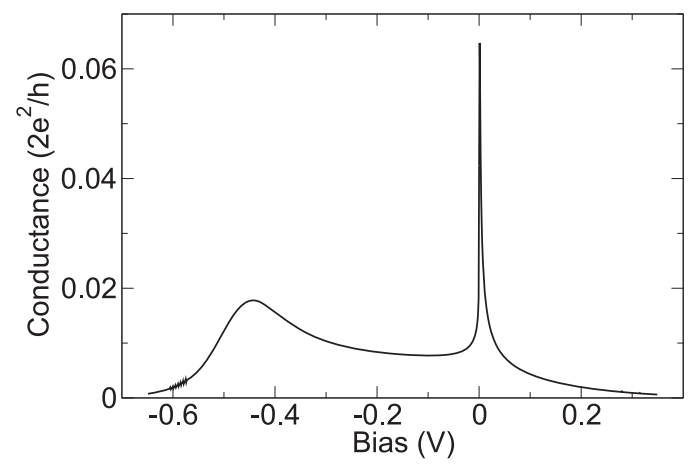

FIG. 7. Conductance computed from the previous transmissions for a bias step of $1 \mathrm{mV}$, Fig. 6 . The difference is a proportionality constant, showing that the spectral function determines the differential conductance in the tunneling regime albeit interference effects between tunneling paths. ${ }^{49,51}$

When the tip is pressed on the atom, a chemical bond forms. At contact $\Gamma_{t} \approx \Gamma_{s}$. We use the same couplings to tip and substrate and compute both spectral functions and differential conductance. Due to the large coupling to the tip, the electronic structure of the adsorbate is not fixed to the substrate, see Fig. 8. In particular, the Kondo peak presents a clear splitting of the order of the applied bias due to the presence of two Fermi levels. ${ }^{21-24,27,28}$ As the bias increases, the spectral function grows more distorted due to the separation of the two Kondo peaks and their reduction due to the bias-induced decoherence of the scattering electrons..$^{22,24-26}$

In the presence of applied bias between electrodes, the Kondo cloud due to one of the substrates can leak into the other substrate leading to decoherence of the Kondo ground state. Using the image of Ref. 22, we can envision the Kondo effect as a coherent spin flip in which an electron coherently hops on and off the impurity. Decoherence takes place if the hopping processes are interrupted. This happens when bias is applied because a new channel opens for the decay of the electron, when the electron hops on the impurity. In other words, the intermediate state associated with the electron in the impurity develops a lifetime that is identified with the bias-induced decoherence rate. ${ }^{22,24-26}$ The decoherence rate ${ }^{24}$ is then the lifetime of the impurity occupied state (or pseudofermion),

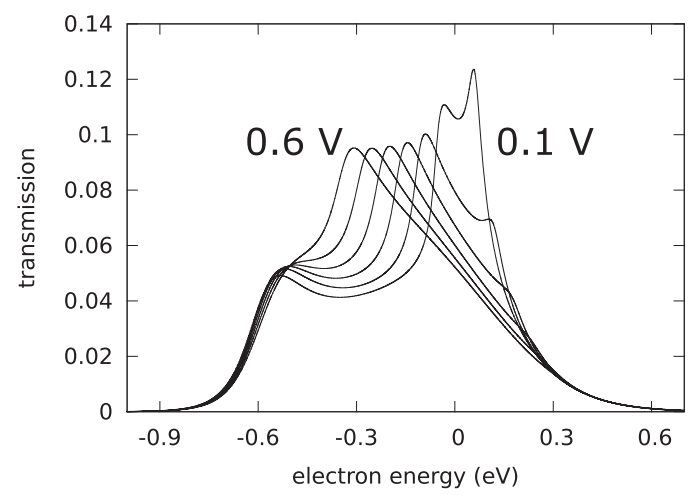

FIG. 8. Transmission curves at contact (equal couplings to both electrodes) for 6 biases from $0.1 \mathrm{~V}$ to $0.6 \mathrm{~V}$, these two extreme values have been marked on the corresponding transmission functions. The characteristic splitting of the Kondo peak is already visible at $0.1 \mathrm{~V}$ since the Kondo temperature is smaller. The splitting clearly follows the bias. Decoherence is also patent in the reduction of the peaks and the disappearance of Kondo structure from the transmission functions. 
given by twice the imaginary part of the self-energy of the occupied-state resolvent. ${ }^{54}$

The differential conductance at contact does not resemble at all the spectral function contrary to the above tunneling case. Since the coupling to the electrodes is symmetric, the conductance has to be symmetric as found in Fig. 9. This clearly does not reflect the spectral function, proportional to the transmissions plotted in Fig. 8. Moreover, the successive contributions of the spectral functions remove any trace of the bias splitting of the Kondo peak in the density of states. Finally, the conductance features a broad symmetric peak.

Experimentally, the sharp structure found in tunneling develops in a featureless broad peak in excellent agreement with the non-equilibrium Kondo differential conductance of the above calculations. Closer inspection to the calculated spectral functions and conductance reveals that the width of the conductance peak is roughly twice the width of the zerobias spectral function. This factor of two is due to the $U \rightarrow \infty$ approximation contained in NCA together with the symmetric condition of equal coupling to both electrodes and is not related to any intrinsic feature of non-equilibrium Kondo physics. Indeed, the spectral function of the NCA equilibrium calculation is very asymmetric due to the $U \rightarrow \infty$ requirement of NCA. Hence, simple symmetrization to obtain a correct differential conductance leads to the factor of two. Only the actual shape of the peak, as bias increases, reflects the appearance of bias-induced decoherence and splitting of the Kondo peak. Figure 9 shows a Frota function fit, Eq. (1), together with the calculated non-equilibrium NCA conductance. As expected, at low-bias, the Frota function is a good fit, while the flatter behavior of NCA conductance at a larger bias signals the appearance of decoherence and non-equilibrium effects. Experimentally then, most features can be extracted from the equilibrium spectral function allowing for the correct number of electrodes in the calculation of the spectral function and of the conductance.

The consequence of this study is that the experimentally determined Kondo temperature does reflect the Kondo temperature of the Co atom due to the increased coupling to its environment. Non-equilibrium effects are then a small correction to the estimated temperatures.

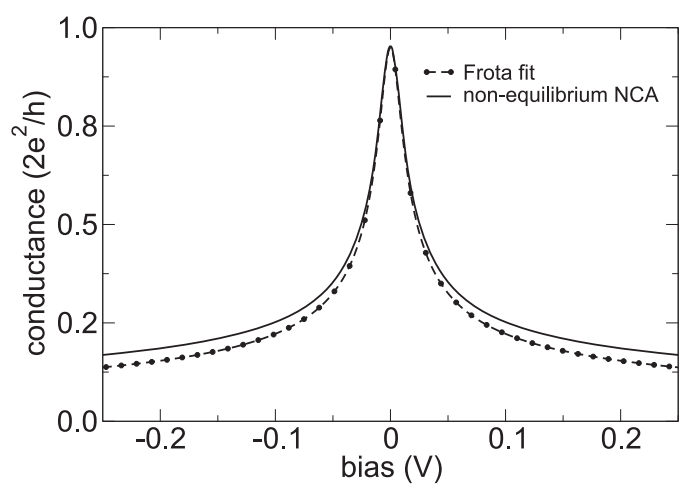

FIG. 9. Conductance computed from a dense grid (steps of $1 \mathrm{mV}$ ) of biasdependent transmissions, Fig. 8. The contact regime leads to a broadened and very symmetric conductance in agreement with the experiment. The dasheddotted line is the Frota function fit, Eq. (1). The fit clearly fails to reproduce the larger-bias behavior of the non-equilibrium calculation.

\section{CONCLUSIONS}

The STM tip can be controllably positioned to create a point contact with a single magnetic atom between tip and sample by adjusting the distance between these two nonmagnetic electrodes. This extraordinary setup permits us to study spin-dependent transport through an atomic constriction with unprecedented accuracy. The junction atom is a Co atom adsorbed on a $\mathrm{Cu}(100)$ surface and contacted by a $\mathrm{Cu}-$ covered STM tip. The differential conductance at low bias shows spin-flip scattering leading to a ZBA identified as the Kondo peak. In tunneling, a characteristic Fano shape reveals that transport takes place through a non-magnetic channel interfering with the Kondo channel of the magnetic impurity. In contact the transport and Kondo channels coalesce and a high-conductance regime leads to a non-equilibrium Kondo peak.

Using DFT and NEGF transport calculations, we can identify the different channels as well as the magnetic structure of the atomic junction. Cobalt maintains its magnetic moment of $2 \mu_{B}$ regardless of the tip-substrate distance, but the transport properties change as the tip approaches the sample. In tunneling, transport proceeds by the $s p$ electron structure of the adsorbate. When the couplings of the atom to tip and substrate equilibrate, new conduction channels open due to the hybridization of the Co $d$-electrons to the tip. At contact one single channel dominates with a $d_{z^{2}}$ character. This orbital possesses the largest overlap with the holding substrate, making it responsible for the Kondo signature. Indeed, despite being a $\mathrm{S}=1$ system, $\mathrm{Co}$ on $\mathrm{Cu}(100)$ is basically a $\mathrm{S}=1 / 2 \mathrm{Kondo}$ system due to the single $d_{z^{2}}$ spin. ${ }^{20,49,50}$

To treat the non-equilibrium Kondo physics when the tip drives the system into the contact regime, we use the nonequilibrium non-crossing approximation. In tunneling, the computed conductance is shown to be proportional to the tunneling spectral function. This is not true in contact, where the spectral function develops two Kondo peaks when the applied bias is larger than the Kondo temperature and the peaks decay by bias-induced decoherence.

Despite this very different regime, the width of the ZBA conductance is fixed by the equilibrium spectral function, reflecting the increase of the Kondo temperature due to the larger couplings to electrodes in the contact regime. Hence, the experimentally obtained widths are indeed proportional to the Kondo temperature of the system as the point contact increases its atomic coordination.

\section{ACKNOWLEDGMENTS}

P.A. and N.L. sincerely thank Jaime Ferrer, Pablo Rivero, and Salva Barraza for providing us with their cobalt pseudopotential. D.J.C. acknowledges the European Union for support under H2020-MSCA-IF-2014 Marie-Curie Individual Fellowship programme Proposal No. 654469. P.A. acknowledges the CCT-Rosario Computational Center. Financial support from CONICET, the Agence Nationale de la Recherche (Grant Nos. ANR-13-BS10-0016, ANR11-LABX-0058 NIE, and ANR-10-LABX-0026 CSC), and MINECO (Grant No. MAT2015-66888-C3-2-R) is gratefully acknowledged. 
${ }^{1}$ W. D. Haas and G. V. D. Berg, Physica 3, 440 (1936).

${ }^{2}$ J. Kondo, Prog. Theor. Phys. 32, 37 (1964).

${ }^{3}$ D. Goldhaber-Gordon, H. Shtrikman, D. Mahaly, D. Abusch-Magder,

U. Meirav, and M. A. Kastner, Nature 391, 156 (1998).

${ }^{4}$ S. M. Cronenwett, T. H. Oosterkamp, and L. P. Kouwenhoven, Science 281, 540 (1998).

${ }^{5}$ M. Reyes-Calvo, J. Fernández-Rossier, J. J. Palacios, D. Jacob, D. Natelson, and C. Untiedt, Nature 458, 1150 (2009).

${ }^{6}$ J. Nygard, D. H. Cobden, and P. E. Lindelof, Nature 408, 342 (2000).

${ }^{7}$ J. Li, W.-D. Schneider, R. Berndt, and B. Delley, Phys. Rev. Lett. 80, 2893 (1998).

${ }^{8}$ V. Madhavan, W. Chen, T. Jamneala, M. F. Crommie, and N. S. Wingreen, Science 280, 567 (1998).

${ }^{9}$ K. Nagaoka, T. Jamneala, M. Grobis, and M. F. Crommie, Phys. Rev. Lett. 88, 077205 (2002).

${ }^{10}$ M. Ternes, A. J. Heinrich, and W.-D. Schneider, J. Phys.: Condens. Matter 21, 053001 (2009).

${ }^{11}$ A. F. Otte, M. Ternes, K. von Bergmann, S. Loth, H. Brune, C. P. Lutz, C. F. Hirjibehedin, and A. J. Heinrich, Nat. Phys. 4, 847 (2008).

${ }^{12}$ H. Pruser, M. Wenderoth, P. E. Dargel, A. Weismann, R. Peters, T. Pruschke, and R. G. Ulbrich, Nat. Phys. 7, 203 (2011).

${ }^{13}$ K. von Bergmann, M. Ternes, S. Loth, C. P. Lutz, and A. J. Heinrich, Phys. Rev. Lett. 114, 076601 (2015).

${ }^{14}$ J. Park, A. N. Pasupathy, J. I. Goldsmith, C. Chang, Y. Yaish, J. R. Petta, M. Rinkoski, J. P. Sethna, H. D. Abruña, P. L. McEuen, and D. C. Ralph, Nature 417, 722 (2002).

${ }^{15}$ U. G. E. Perera, H. J. Kulik, V. Iancu, L. G. G. V. Dias da Silva, S. E. Ulloa, N. Marzari, and S.-W. Hla, Phys. Rev. Lett. 105, 106601 (2010).

${ }^{16}$ M. Ormaza, R. Robles, N. Bachellier, P. Abufager, N. Lorente, and L. Limot, Nano Lett. 16, 588 (2016).

${ }^{17}$ J. Kröger, N. Néel, and L. Limot, J. Phys.: Condens. Matter 20, 223001 (2008).

${ }^{18}$ N. Néel, J. Kröger, L. Limot, K. Palotas, W. A. Hofer, and R. Berndt, Phys. Rev. Lett. 98, 016801 (2007).

${ }^{19}$ D.-J. Choi, M. V. Rastei, P. Simon, and L. Limot, Phys. Rev. Lett. 108, 266803 (2012).

${ }^{20}$ D.-J. Choi, S. Guissart, M. Ormaza, N. Bachellier, O. Bengone, P. Simon, and L. Limot, Nano Lett. 16, 6298 (2016).

${ }^{21}$ S. Hershfield, J. H. Davies, and J. W. Wilkins, Phys. Rev. Lett. 67, 3720 (1991).

${ }^{22}$ Y. Meir, N. S. Wingreen, and P. A. Lee, Phys. Rev. Lett. 70, 2601 (1993).

${ }^{23}$ M. H. Hettler, J. Kroha, and S. Hershfield, Phys. Rev. B 58, 5649 (1998).

${ }^{24}$ A. Rosch, J. Kroha, and P. Wölfle, Phys. Rev. Lett. 87, 156802 (2001).

${ }^{25}$ R. C. Monreal and F. Flores, Phys. Rev. B 72, 195105 (2005).
${ }^{26}$ R. Van Roermund, S.-y. Shiau, and M. Lavagna, Phys. Rev. B 81, 165115 (2010).

${ }^{27}$ P. R. Bas and A. A. Aligia, J. Phys.: Condens. Matter 22, 025602 (2009).

${ }^{28}$ G. Cohen, E. Gull, D. R. Reichman, and A. J. Millis, Phys. Rev. Lett. 112, 146802 (2014).

${ }^{29}$ L. Limot, J. Kröger, R. Berndt, A. Garcia-Lekue, and W. A. Hofer, Phys. Rev. Lett. 94, 126102 (2005).

${ }^{30}$ M. Ternes, C. González, C. P. Lutz, P. Hapala, F. J. Giessibl, P. Jelínek, and A. J. Heinrich, Phys. Rev. Lett. 106, 016802 (2011).

${ }^{31}$ N. Néel, J. Kröger, and R. Berndt, Phys. Rev. Lett. 102, 086805 (2009).

${ }^{32}$ K. Tao, I. Rungger, S. Sanvito, and V. S. Stepanyuk, Phys. Rev. B 82, 085412 (2010).

${ }^{33}$ H. O. Frota and L. N. Oliveira, Phys. Rev. B 33, 7871 (1986).

${ }^{34}$ N. Agraï, C. Untiedt, G. Rubio-Bollinger, and S. Vieira, Phys. Rev. Lett. 88, 216803 (2002).

${ }^{35}$ T. Frederiksen, M. Paulsson, M. Brandbyge, and A.-P. Jauho, Phys. Rev. B 75, 205413 (2007).

${ }^{36}$ J. P. Perdew, K. Burke, and M. Ernzerhof, Phys. Rev. Lett. 77, 3865 (1996).

${ }^{37}$ G. Kresse and J. Hafner, Phys. Rev. B 47, 558 (1993).

${ }^{38}$ G. Kresse and J. Hafner, Phys. Rev. B 48, 13115 (1993).

${ }^{39}$ G. Kresse and J. Furthmüller, Comput. Mater. Sci. 6, 15 (1996).

${ }^{40}$ G. Kresse and J. Furthmüller, Phys. Rev. B 54, 11169 (1996).

${ }^{41}$ G. Kresse and D. Joubert, Phys. Rev. B 59, 1758 (1999).

${ }^{42}$ J. Hafner, J. Comput. Chem. 29, 2044 (2008).

${ }^{43}$ J. M. Soler, E. Artacho, J. D. Gale, A. García, J. Junquera, P. Ordejón, and D. Sánchez-Portal, J. Phys.: Condens. Matter 14, 2745 (2002).

${ }^{44}$ E. Artacho, E. Anglada, O. Diéguez, J. D. Gale, A. García, J. Junquera, R. M. Martin, P. Ordejn, J. M. Pruneda, D. Snchez-Portal et al., J. Phys.: Condens. Matter 20, 064208 (2008).

${ }^{45}$ M. Brandbyge, J. Mozos, P. Ordejon, J. Taylor, and K. Stokbro, Phys. Rev. B 65, 165401 (2002).

${ }^{46}$ P. Abufager, R. Robles, and N. Lorente, J. Phys. Chem. C 119, 12119 (2015).

${ }^{47}$ M. Polok, D. V. Fedorov, A. Bagrets, P. Zahn, and I. Mertig, Phys. Rev. B 83, 245426 (2011).

${ }^{48}$ B. Surer, M. Troyer, P. Werner, T. O. Wehling, A. M. Läuchli, A. Wilhelm, and A. I. Lichtenstein, Phys. Rev. B 85, 085114 (2012).

${ }^{49}$ P. P. Baruselli, R. Requist, A. Smogunov, M. Fabrizio, and E. Tosatti, Phys. Rev. B 92, 045119 (2015).

${ }^{50}$ D. Jacob, J. Phys.: Condens. Matter 27, 245606 (2015).

${ }^{51}$ S. Frank and D. Jacob, Phys. Rev. B 92, 235127 (2015).

${ }^{52}$ H. T. Dang, M. dos Santos Dias, A. Liebsch, and S. Lounis, Phys. Rev. B 93, 115123 (2016).

${ }^{53}$ Y. Meir and N. S. Wingreen, Phys. Rev. Lett. 68, 2512 (1992).

${ }^{54}$ R. Korytár and N. Lorente, J. Phys.: Condens. Matter 23, 355009 (2011). 